\title{
FINITELY ADDITIVE CORRESPONDENCES
}

\author{
ACHILLE BASILE
}

(Communicated by Andrew M. Bruckner)

\begin{abstract}
Let $X$ be a locally convex space. Finitely additive $X$-valued correspondences are investigated, and exhaustive, weakly compact-valued ones are shown to be rich in selections. If, in addition, $X$ is metrizable, then one of such selections is a control. The range of nonatomic correspondences is also studied.
\end{abstract}

\section{INTRODUCTION}

This paper deals with finitely additive correspondences, namely, with mappings $\Phi: \mathscr{F} \rightarrow \mathscr{P}_{0}(X)$ (where $\mathscr{F}$ is a Boolean algebra and $\mathscr{P}_{0}(X)$ is the collection of nonempty subsets of the range space $X$ ) for which the following two conditions are satisfied: $\Phi(0)=\{0\}$ and, for disjoint $a, b \in \mathscr{F}$, $\Phi(a \vee b)=\Phi(a)+\Phi(b)$. To make such conditions meaningful, we set $X_{1}+X_{2}=$ $\left\{x_{1}+x_{2}: x_{i} \in X_{i}\right\}$ and may assume that $X$ is some abelian semigroup with a neutral element. The deepest results, however, will concern the case of a locally convex linear space $X$. They will be obtained by specializing results relative to the case that the range space is some uniform semigroup.

The interest in studying additive correspondences originates from the theory of general economic equilibrium, and already the paper [V] by Vind points out two of the main directions of investigation for additive correspondences, richness in selections and geometric structure of the range $R \Phi=\bigcup\{\Phi(b): b \in$ $\mathscr{F}$ \}. A third main direction deals with the integral representation of additive correspondences. Debreu [D], Debreu and Schmeidler [DS], and Hildenbrand $[\mathrm{H}]$ can serve as motivating and initial references. Even if these directions are not independent, we shall deal only with the first two.

In [V] a Lyapunov-type theorem is proved for the net trade correspondence of an exchange economy assuming that such correspondence $\left(R^{n}\right.$-valued of course) is countably additive, has no atoms and is rich in selections (see Definition 1). Deeper results were later obtained by Schmeidler [Sc] and Artstein [A], always for countably additive $R^{n}$-valued correspondences. In particular, [A] contains contributions to any of the directions indicated above, and they are,

Received by the editors June 29, 1992 and, in revised form, October 28, 1992; presented to the "Fifth Capri Meeting on Real Analysis and Measure Theory", Isola di Capri, September 7-11, 1992.

1991 Mathematics Subject Classification. Primary 28B20; Secondary 28B10, 90A14, 46G10. 
in their setting, in a sense definitive. Further progress is due to the work by Schmeidler [Sc1], Godet-Thobie [GT, GT1], Drewnowski [Dr1], Costé [C, C1, C2], Pallu de LaBarriere [P], and Le Van Tu [L] that considered, with the exception of Drewnowski, countably additive correspondences taking values in infinite-dimensional spaces.

The reason to deal with correspondences that are simply finitely rather than countably additive still bases on applications to the economic theory. Indeed Armstrong and Richter [AR] gave more realism to the model of a large economy, letting it be founded on the assumption of finite additivity of the underlying measure space of agents. As a consequence, in [B1] is extended the ArmstrongRichter framework from pure exchange to coalition production economies on the base of results for closed-valued finitely additive correspondences extending some of those in [A]. The present paper enlarges and deepens the mathematical part of [B1]. Its main results generalize several of those already known in the literature for the countably additive setting.

\section{SEMigRoup-VALUED CORRESPONDENCES}

In this section $(X,+, 0, \mathscr{U})$ is a separated uniform abelian semigroup, and, unless it is differently stated, $\Phi$ is an $X$-valued finitely additive correspondence over the Boolean algebra $\mathscr{F}$.

Definition 1. Let $S$ be the set of all finitely additive selections of $\Phi$. We say that $\Phi$ is rich in selections if $S$ is nonempty and, for all $a \in \mathscr{F}, \Phi(a)=$ $\{\mu(a): \mu \in S\}$.

Proposition 1. If $\mathscr{F}$ is finite, then $\Phi$ is rich in selections.

Proof. Assume $\mathscr{F}$ is the family of subsets of $\{1,2, \ldots, p\}$ for some $p$. If $a$ is a nonempty subset of $\{1,2, \ldots, p\}$ then $\Phi(a)=\sum_{i \in a} \Phi(\{i\})$. Therefore, if $x \in \Phi(a)$, there are $x_{i} \in \Phi(\{i\})$ such that $x=\sum_{i \in a} x_{i}$. Choosing, for $i \notin a$, arbitrary $x_{i} \in \Phi(\{i\})$ we have that the function $\mu(b):=\sum_{i \in b} x_{i}, b \in \mathscr{F}$, is a finitely additive selection of $\mathscr{F}$ taking value $x$ on $a$.

It is well known (see [W]) that the uniformity $\mathscr{U}$ of $X$ is generated by a family $\left\{p_{i}: i \in I\right\}$ of semi-invariant pseudometrics (namely, pseudometrics for which $p_{i}(a+c, b+c) \leq p_{i}(a, b)$ holds for all $\left.a, b, c \in X\right)$. The so-called Hausdorff extension of $p_{i}$ to $\mathscr{P}_{0}(X)$ [KT, Chapter IV] defined by

$$
P_{i}(A, B):=\inf \left\{\varepsilon>0: A \subseteq B_{p_{i}}[\varepsilon] \text { and } B \subseteq A_{p_{i}}[\varepsilon]\right\}
$$

(where $A_{p_{i}}[\varepsilon]=\bigcup_{y \in A}\left\{x: p_{i}(x, y) \leq \varepsilon\right\}$ is also a semi-invariant pseudometric. Therefore, the uniformity $\mathscr{U}^{*}$ generated by $\left\{P_{i}: i \in I\right\}$ makes $\left(\mathscr{P}_{0}(X),+,\{0\}\right)$ a (nonseparated) uniform semigroup. The uniformity $\mathscr{U}^{*}$ is called the Hausdorf uniformity.

Let us denote by $\mathscr{C}(X)$ the subset of $\mathscr{P}_{0}(X)$ consisting of closed sets. Defining $A^{*}+B=\operatorname{cl}(A+B)$ and relativizing the Hausdorff uniformity $\mathscr{U}^{*}$ of $\mathscr{P}_{0}(X)$ to $\mathscr{C}(X)$, we get the separated semigroup $(\mathscr{C}(X), \stackrel{*}{+})$ which is a model for the separated uniform semigroup associated with $\mathscr{P}_{0}(X)$. For a complete metrizable $X$, the metrizable semigroup $\mathscr{C}(x)$ is also complete [KT, Theorem 4.3.8]. We shall call + -finitely additive a correspondence $\Phi$ (not necessarily finitely additive) when the usual additivity is relaxed to $\operatorname{cl}(\Phi(a \vee b))=\Phi(a) \stackrel{*}{+} \Phi(b)$. 
It follows after the above remarks, that a finitely additive correspondence (or one which is closed valued and $\stackrel{*}{+}$-finitely additive) can be seen as an ordinary content ( $=$ finitely additive function) with values in a uniform semigroup. Consequently concepts and results available for such contents are automatically at our disposal. For example, as concepts that will be of interest for us we indicate

(a) $\Gamma(\Phi)$ is a $F N$-topology (see [Dr, W1]) generated by $\Phi$ over the Boolean algebra $\mathscr{F}$. The 0 -neighbourhood base of $\Gamma(\Phi)$ is given by

$$
\omega(V):=\left\{b \in \mathscr{F}: \bigcup_{a \leq b, a \in \mathscr{F}} \Phi(a) \subseteq V\right\}
$$

letting $V$ run over a neighbourhood base of $0 \in X$.

(b) The exhaustivity, or s-boundedness, of $\Phi$ (equivalently of $\Gamma(\Phi)$ ) is: whenever $a_{n} \in \mathscr{F}$ is a disjoint sequence, we have that, for any $V$ in a neighbourhood base of $0 \in X$, eventually $\Phi\left(a_{n}\right) \subseteq V$.

For other concepts here not explicitly recalled we refer to [DR, W1, W2]. A sample of results that are special cases of what is known for semigroup-valued contents is obtained by taking those in [Pr], in [Z], and some (not the main) in [Drl]. The idea of transferring results for functions to correspondences by thinking the latter as functions in special spaces is quite standard, even in the case of additive correspondences. The use of the Minkowsi-RadstromHormander Theorem that permits us to transform (vector-valued) additive correspondences into vector measures goes back to [D]. For their nature, however, the results that we will be dealing with seem not to be mere restatements of known theorems for additive function.

Theorem 1. Assume $R \Phi$ is relatively compact and $\Phi$ is closed valued. Then

(1) $\Phi$ is rich in selections, and

(2) $S$ is a compact subset of the space $X^{\mathscr{F}}$ endowed with the pointwise convergence topology.

Proof. (1) It is enough to prove that for any $x \in \Phi(e)$ (let $e$ be the unit of $\mathscr{F})$ there is a $\mu \in S$ such that $\mu(e)=x$. Let $\mathscr{F}$ be a finite subalgebra of $\mathscr{F}$, and set $\sigma\left(\mathscr{F}_{0}\right)=\left\{\mu \in(\overline{R \Phi})^{\mathscr{F}}: \mu(e)=x ; \mu_{\mid \mathscr{S}_{0}}\right.$ is a finitely additive selection of $\left.\Phi_{\mid \mathscr{F}_{0}}\right\}$. Because of Proposition 1, $\sigma\left(\mathscr{F}_{0}\right)$ is nonempty. Moreover, since $\Phi$ is closed valued, $\sigma\left(\mathscr{F}_{0}\right)$ is a closed subset (with respect to the pointwise convergence topology) of $(\overline{R \Phi})^{\mathscr{F}}$. On the other hand the family $\left\{\sigma\left(\mathscr{F}_{0}\right)\right.$ : $\mathscr{F}$ is a finite subalgebra of $\mathscr{F}\}$ has the finite intersection property, so the compactness of $(\overline{R \Phi})^{\mathscr{F}}$ says that the intersection $\bigcap_{\mathscr{F}_{0}} \sigma(\mathscr{F})$ of the family is nonempty. A function $\mu$ belonging to such an intersection is in $S$, and $\mu(e)=$ $x$.

(2) Note that $S$ is a closed subset of $X^{\mathscr{F}}$ and $S \subset(\overline{R \Phi})^{\mathscr{F}}$.

It will be useful to observe that by definition $S$ is equiabsolutely continuous with respect to $\Gamma(\Phi)$.

Theorem 2. Let $X$ be metrizable and complete. Assume $\Phi: \mathscr{F} \rightarrow \mathscr{C}(X)$ is $\stackrel{*}{+}$ finitely additive, rich in selections, and exhaustive. Then there exists a selection $\gamma$ of $\Phi$ such that $\Gamma(\gamma)=\Gamma(\Phi)$. 
Proof. We shall proceed by proving

(a) there exists a content $\gamma$ such that $\Gamma(\gamma)=\Gamma(S)$,

(b) $\Gamma(\Phi) \subseteq \Gamma(\gamma)$, and

(c) $\gamma \in S$.

The proof of (a) is based on [B, Lemma 1], therefore, notation similar to that of [B] is introduced. Let $\xi$ be the supremum of all exhaustive $F N$-topologies on $\mathscr{F}$, and let $(\widetilde{\mathscr{F}}, \tilde{\xi})$ be the completion of $(\mathscr{F}, \xi)$. Because of the exhaustivity of $\Phi$, the content $\Phi: \mathscr{F} \rightarrow(\mathscr{C}, \stackrel{*}{+})$ can be extended by $\tilde{\xi}$-continuity determining the content $\widetilde{\boldsymbol{\Phi}}: \widetilde{F} \rightarrow(\mathscr{C}, \stackrel{*}{+})$. We do the same for any $\mu \in S$, and get the selection $\tilde{\mu}: \widetilde{\mathscr{F}} \rightarrow X$ of $\Phi$. Set $\tilde{S}=\{\tilde{\mu}: \mu \in S\}$. By [W1, Corollary (2.3)], $\Gamma(S) \subseteq \Gamma(\Phi)$ tells us that $\Gamma(S)$ is pseudometrizable; therefore, we can choose countably many $\mu_{i} \in S$ in such a way that $\Gamma\left(\mu_{1}, \ldots, \mu_{n}, \ldots\right)=\Gamma(S)$. Denote by $N(\phi)$ the ideal of $\phi$-null elements of $\mathscr{F}$ when $\phi$ is a $F N$-topology (or a content).

Let us define elements of $\widetilde{\mathscr{F}}$ by

$$
\begin{gathered}
a_{S}:=e \backslash \max N(\Gamma(\tilde{S})), \quad a_{\mu_{i}}:=e \backslash \max N\left(\tilde{\mu}_{i}\right), \quad a_{1}:=a_{\mu_{1}}, \\
a_{n+1}:=a_{\mu_{n+1}} \backslash\left(a_{\mu_{1}} \vee \cdots \vee a_{\mu_{n}}\right) .
\end{gathered}
$$

Note that in $(\widetilde{\mathscr{F}}, \tilde{\xi})$

$$
a_{S}=\lim _{n} \bigvee_{i=1}^{n} a_{i}=\bigvee_{n=1}^{\infty} a_{n}
$$

Since $\Gamma(\tilde{\Phi}) \subseteq \tilde{\xi}$, the sequence $\bigvee_{i=1}^{n} a_{i}$ is also $\Gamma(\widetilde{\Phi})$-convergent, and consequently the series

$$
\tilde{\gamma}(b):=\sum_{i=1}^{\infty} \tilde{\mu}_{i}\left(b \wedge a_{i}\right)
$$

is uniformly convergent over $\widetilde{F}$.

Indeed, let $V$ be a neighbourhood of $0 \in X$ and $h$ an index such that

$$
q>p \geq h, \quad b \in \widetilde{\mathscr{F}}, \quad b \leq \bigvee_{p \leq i<q} a_{i} \Rightarrow \widetilde{\Phi}(b) \subseteq V .
$$

We have

$$
\sum_{p \leq i<q} \tilde{\mu}_{i}\left(b \wedge a_{i}\right) \subseteq \tilde{\Phi}\left(b \wedge \bigvee_{p \leq i<q} a_{i}\right) \subseteq V
$$

Now [B, Lemma 1] says that $\Gamma(\tilde{\gamma})=\Gamma(\tilde{S})$ and the desired control for $S$ is $\gamma:=\tilde{\gamma}_{\mid \mathscr{F}}$.

To prove (b) observe that $S$ is uniformly exhaustive and therefore also equiabsolutely continuous with respect to $\gamma$. For a given neighbourhood $V$ of $0 \in X$ let $W$ be another of such neighbourhoods for which

$$
\{b \in \mathscr{F}: \gamma(\mathscr{F} \wedge b) \subseteq W\} \subseteq \bigcap_{\mu \in S}\{b \in \mathscr{F}: \mu(\mathscr{F} \wedge b) \subseteq V\}
$$


Because $\Phi$ is rich in selections, we have

$$
\{b \in \mathscr{F}: \gamma(\mathscr{F} \wedge b) \subseteq W\} \subseteq\left\{b \in \mathscr{F}: \bigcup_{a \leq b, a \in \mathscr{F}} \Phi(a) \subseteq V\right\},
$$

which shows (b).

Finally, to get (c), note that $\Gamma(\Phi) \subseteq \Gamma(\gamma)$ implies $\Gamma(\tilde{\Phi}) \subseteq \Gamma(\tilde{\gamma})=\Gamma(\widetilde{S})$, and therefore, by (1), for $b \in \mathscr{F}$ we have

$$
b \backslash \bigvee_{i=1}^{n} a_{i} \stackrel{\tilde{\xi}}{\rightarrow} b \backslash a_{S} \in N(\Gamma(\tilde{S})) \subseteq N(\Gamma(\widetilde{\Phi}))
$$

and

$$
b \wedge \bigvee_{i=1}^{n} a_{i} \stackrel{\Gamma(\widetilde{\Phi})}{\rightarrow} b
$$

It follows that

$$
\widetilde{\boldsymbol{\Phi}}\left(b \wedge \bigvee_{i=1}^{n} a_{i}\right) \stackrel{\left(\mathscr{C}, \mathscr{U} *^{*}\right)}{\longrightarrow} \widetilde{\Phi}(b)
$$

The latter, together with

$$
\sum_{i=1}^{n} \tilde{\mu}_{i}\left(b \wedge a_{i}\right) \in \widetilde{\boldsymbol{\Phi}}\left(b \wedge \bigvee_{i=1}^{n} a_{i}\right)
$$

gives $\tilde{\gamma}(b) \in \widetilde{\Phi}(b)$.

We now move our attention to the range of a nonatomic $\Phi$. For this aim we will assume that $\mathscr{F}$ has the Seever property (see [Bh]); namely, for any pair of sequences $a_{n}, b_{n} \in \mathscr{F}$ with $a_{n} \leq a_{n+1} \leq b_{m+1} \leq b_{m}$, there exists $c \in \mathscr{F}$ such that $a_{n} \leq c \leq b_{m}$ holds for all natural numbers $n, m$.

Let us recall that the correspondence $\Phi$ is said to be nonatomic when $(\Gamma(\Phi)$ is, i.e.) for every neighbourhood $V$ of $0 \in X$ there are finitely many disjoint $b_{i} \in \mathscr{F}$ such that the unit $e$ of $\mathscr{F}$ is their supremum and $\Phi(a) \subseteq V$ whenever $a \leq b_{i}, a \in \mathscr{F}$. Then it is plain after [VW, Proposition 1.1] (which holds with the weaker assumption of $\mathscr{F}$ with the Seever Property) that for a pseudometrizable nonatomic $\Phi$ we have arcwise connectness of $(\mathscr{F}, \Gamma(\Phi))$ and consequently the following result holds.

Proposition 2. Assume $\mathscr{F}$ has Seever property. Let $\Phi$ be rich in selections, nonatomic, and with a metrizable range. Then the values and the range of $\Phi$ are arcwise connected.

Proof. Since $R \Phi$ is a metrizable part of $X$, we derive that $\Gamma(\Phi)$ is pseudometrizable. $R \Phi$ is arcwise connected because the richness in selections of $\Phi$ entails $R \Phi=\bigcup_{\mu \in S} R \mu$ and each $R \mu$ is arcwise connected, being a continuous image of the arcwise connected space $(\mathscr{F}, \Gamma(\Phi))$.

Now let $b \in \mathscr{F} \backslash N(\Gamma(\Phi))$ and $x, y \in \Phi(b)$. Let $\alpha$ be as in [VW, Proposition 1.1]. Because $\Phi$ is rich in selections, we find $\mu_{1}$ and $\mu_{2}$ in $S$ such that $\mu_{1}(b)=x$ and $\mu_{2}(b)=y$. Set $\beta(t):=\mu_{1}(\alpha(t))+\mu_{2}(b \backslash \alpha(t))$ for $t \in[0,1]$. $\beta$ is an arc (continuous) connecting $x$ and $y$ without leaving $\Phi(b)$. 


\section{VECTOR-VALUED CORRESPONDENCES}

Let $\Phi$ be a correspondence from the Boolean algebra $\mathscr{F}$ to the nonempty subsets of a separated locally convex linear space $X$. Let us fix the notation $\left\langle A, x^{\prime}\right\rangle:=\sup \left\{\left\langle x, x^{\prime}\right\rangle: x \in A\right\}$ for the support function of a subset $A$ of $X \quad\left(x^{\prime} \in X^{\prime}=\right.$ the topological dual of $\left.X\right)$. $\mathscr{W}(X)$ denotes the part of $\mathscr{C}(X)$ consisting of weakly compact subsets of $X$. We shall shorten the symbol $\bar{A}^{\sigma\left(X, X^{\prime}\right)}$-denoting the closure of $A$ with respect to the weak topology $\sigma\left(X, X^{\prime}\right)$-by writing $\bar{A}^{\sigma}$. Also, $\mathscr{W}$ will be used to shorten $\mathscr{W}(X)$.

Trivially if $A, B \in \mathscr{W}$ then $A+B \in \mathscr{W}$, and therefore $A \stackrel{*}{+} B=A+B$. Moreover, if $\Phi$ is $\stackrel{*}{+}$-finitely additive and relatively weakly compact valued, by taking the weak closure of the values of $\Phi$, we have the correspondence $\bar{\Phi}^{\sigma}: \mathscr{F} \rightarrow \mathscr{W}$ for which we note (if $a$ and $b$ are disjoint)

$$
\begin{aligned}
\overline{\boldsymbol{\Phi}}^{\sigma}(a \vee b) & =\overline{\boldsymbol{\Phi}(a)+*}+\boldsymbol{\Phi}(b)^{\sigma}=\overline{(\mathrm{cl}[\Phi(a)+\Phi(b)])}^{\sigma} \\
& =\overline{\boldsymbol{\Phi}(a)+\Phi(b)}^{\sigma}={\overline{\overline{\boldsymbol{\Phi}}^{\sigma}(a)+\overline{\boldsymbol{\Phi}}^{\sigma}(b)}}^{\sigma}=\overline{\boldsymbol{\Phi}}^{\sigma}(a)+\overline{\boldsymbol{\Phi}}^{\sigma}(b),
\end{aligned}
$$

namely, the finite additivity. Moreover, if $\Phi$ is assumed to be exhaustive, clearly $\overline{\boldsymbol{\Phi}}^{\sigma}$ is also exhaustive.

The notation $C_{r}$ will be reserved for the closed ball of radius $r$ (centered at the origin) in the case that $X$ is a normed space.

As in [T], by using that (see [J]) a weakly closed subset of a Banach space is weakly compact if and only if each continuous linear form attains its supremum on it, we shall obtain in Theorem 3 the relative weak compactness of the range of an exhaustive $\Phi$ generalizing what is known for the single-valued case [DU, Chapter 1]. First note the following lemma.

Lemma 1. Let $X$ be a Banach space and the correspondence $\Phi$ be relatively weakly compact-valued. Assume further that for any $x^{\prime} \in X^{\prime}$ the set function $\left\langle\Phi(\cdot), x^{\prime}\right\rangle: \mathscr{F} \rightarrow R$ is a content admitting a Hahn decomposition. It follows that $R \Phi$ is relatively weakly compact.

Proof. We have to show that any $x^{\prime} \in X^{\prime}$ takes maximum over $\overline{R \Phi}^{\sigma}$. Let $\left\{e^{+}, e^{-}\right\}$be the Hahn decomposition of $\left\langle\Phi(\cdot), x^{\prime}\right\rangle$, and note that $\left\langle\overline{R \Phi}^{\sigma}, x^{\prime}\right\rangle=$ $\left\langle R \Phi, x^{\prime}\right\rangle=\sup \left\{\left\langle\Phi(b), x^{\prime}\right\rangle: b \in \mathscr{F}\right\}=\left\langle\Phi\left(e^{+}\right), x^{\prime}\right\rangle=\left\langle\overline{\Phi\left(e^{+}\right)}{ }^{\sigma}, x^{\prime}\right\rangle$. Being $\overline{\Phi\left(e^{+}\right)}$weakly compact, $\left\langle{\overline{\Phi\left(e^{+}\right)}}^{\sigma}, x^{\prime}\right\rangle$ can be written as $\left\langle x, x^{\prime}\right\rangle$ where $x \in$ ${\overline{\Phi\left(e^{+}\right)}}^{\sigma} \subseteq \overline{R \Phi}^{\sigma}$.

Lemma 2 [Gr]. Let $W$ be a subset of the Banach space $X . W$ is relatively weakly compact if for any positive $r$ a weakly compact set $W_{r}$ exists such that $W \subseteq W_{r}+C_{r}$.

Theorem 3. Assume that $X$ is a complete separated locally convex space and $\Phi$ is a+-finitely additive and relatively weakly compact-valued correspondence on the Boolean algebra $\mathscr{F}$. Then if $\Phi$ is exhaustive its range is relatively weakly compact.

Proof. As we show later, the proof can be reduced to the case of Banach spaces. Therefore, assume now that $X$ is such a space.

Let us consider the finitely additive and exhaustive $\bar{\Phi}^{\sigma}: \mathscr{F} \rightarrow \mathscr{W}$. As in the proof of Theorem 2 let $\widetilde{\Phi}: \widetilde{F} \rightarrow(\mathscr{C}, \stackrel{*}{+})$ be the content extending $\overline{\boldsymbol{\Phi}}^{\sigma}$ 
by $\tilde{\xi}$-continuity. The values of $\tilde{\Phi}$ are also relatively weakly compact. For if $b \in \widetilde{\mathscr{F}}$, then $\widetilde{\Phi}(b)$ is the limit (with respect to the Hausdorff metric) in $\mathscr{C}$ of a net $\bar{\Phi}^{\sigma}\left(b_{\lambda}\right)$ with $b_{\lambda} \in \mathscr{F}$. By definition, given $r$, we have $\lambda$ such that $\widetilde{\Phi}(b) \subseteq \overline{\boldsymbol{\Phi}}^{\sigma}\left(b_{\lambda}\right)+C_{r}$, and Lemma 2 gives the relative weak compactness of $\widetilde{\Phi}(b)$. Plainly, for any $x^{\prime} \in X^{\prime}$, the function $\left\langle\widetilde{\Phi}(\cdot), x^{\prime}\right\rangle$ is an ordinary measure $(\widetilde{F}$ is a complete Boolean algebra, and $\widetilde{\Phi}$ is $\tau$-smooth; see [W1]). Therefore, by Lemma $1, R \widetilde{\Phi}$ is relatively weakly compact. Now observe that $R \Phi \subseteq \bigcup_{b \in \mathscr{F}} \bar{\Phi}^{\sigma}(b) \subseteq R \widetilde{\Phi}$. With this, the proof for the Banach space case is finished.

Assume now that $X$ is any locally convex space. $X$ is embedded in a product $E=\prod_{i \in I} B_{i}$ of Banach spaces $B_{i}$. Note that by [S, Theorem 4.3] the weak topology $\sigma\left(E, E^{\prime}\right)$ is simply the product of the weak topologies $\sigma\left(B_{i}, B_{i}^{\prime}\right)$. Consequently the projection mapping $\pi_{i}: E \rightarrow B_{i}$ is also $\sigma\left(E, E^{\prime}\right)-\sigma\left(B_{i}, B_{i}^{\prime}\right)$ continuous.

Set $\Phi_{i}(b):=\pi_{i}\left(\bar{\Phi}^{\sigma}(b)\right)$. Since $\bar{\Phi}^{\sigma}$ is finitely additive and exhaustive, $\Phi_{i}$ is also so. $\bar{\Phi}^{\sigma}(b)$ is $\sigma\left(X, X^{\prime}\right)$-compact, but $\sigma\left(X, X^{\prime}\right)$ is $\sigma\left(E, E^{\prime}\right)$ relativized to $X$, so $\bar{\Phi}^{\sigma}(b)$ is $\sigma\left(E, E^{\prime}\right)$-compact and, by the continuity of $\pi_{i}$, we have the $\sigma\left(B_{i}, B_{i}^{\prime}\right)$-compactness of $\Phi_{i}(b)$. We can apply what was already proved for Banach spaces, deducing the relative $\sigma\left(B_{i}, B_{i}^{\prime}\right)$-compactness of the range of $\Phi_{i}$. Now

$$
R \Phi \subseteq R \bar{\Phi}^{\sigma} \subseteq \prod_{i \in I} R \Phi_{i} \subseteq \bar{\prod}_{i \in I} R \Phi_{i}^{\sigma\left(E, E^{\prime}\right)} \subseteq \prod_{i \in I}{\overline{R \Phi_{i}}}^{\sigma\left(B_{i}, B_{i}^{\prime}\right)},
$$

so by the Tichonoff theorem $R \Phi$ is relatively $\sigma\left(E, E^{\prime}\right)$-compact. Finally,

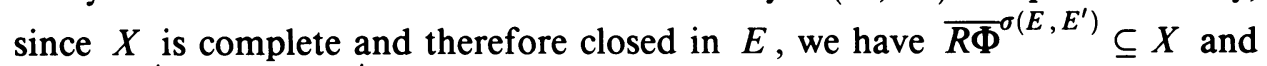
$\overline{R \Phi}^{\sigma\left(X, X^{\prime}\right)}=\overline{R \Phi}^{\sigma\left(E, E^{\prime}\right)}$ is $\sigma\left(X, X^{\prime}\right)$-compact.

Theorem 4. Let $X$ be a separated locally convex space. Assume $\Phi$ is finitely additive and weakly compact-valued. Then $\Phi$ is rich in selections if one of the two following conditions holds:

(a) $\Phi$ is exhaustive.

(b) $\mathscr{F}$ is $\sigma$-complete, and for any $x^{\prime} \in X^{\prime}$ the map $\left\langle\Phi(\cdot), x^{\prime}\right\rangle$ is a realvalued measure.

Proof. We may assume $X$ is complete. If (a) holds then by Theorem 3 the range of $\Phi$ is relatively weakly compact. In the case (b) the same result is reached via Lemma 1, once noted that the assumption that $X$ is Banach can be removed by assuming $X$ locally convex and complete.

Now apply Theorem 1 , treating the values of $\Phi$ as subsets of $\left(X, \sigma\left(X, X^{\prime}\right)\right)$.

Remark. The existence of a selection for a finitely additive, exhaustive, weakly compact-valued $\Phi$ was already stated in [Dr1, Corollary 8.7]; evidently our Theorem 4 (a) refines it. Part (b) of Theorem 4 improves [GT1, Theorem 1], relaxing compactness of values to weak compactness. An improved version of [Dr1, Corollary 8.11] also follows from Theorem 4.

The very significant case of a metrizable range space in [Dr, Theorem 8.12] comes from 
Theorem 5. Let $X$ be a metrizable locally convex space. Assume $\Phi$ is finitely additive, exhaustive, and weakly compact valued. Then we find $\gamma \in S$ such that $\Gamma(\gamma)=\Gamma(\Phi)$.

Proof. We may assume $X$ is complete. By Theorem $4 \Phi$ is rich in selections so Theorem 2 can be applied.

Finally we again turn our attention to the range of $\Phi$ by registering the following statements.

By means of Proposition 2 and Theorem 4 we get

Proposition 3. Assume $\mathscr{F}$ has Seever property and $X$ is a Banach space. If $\Phi$ is finitely additive, nonatomic, exhaustive, and weakly compact-valued, then its values and its range are arcwise connected.

In the following proposition, (1) is [B1, Theorem $5(\mathrm{a}),(\mathrm{b})],(2)$ is in $[\mathrm{AB}]$, and $(3)$ is trivial.

Proposition 4. Assume $X=R^{n}$ and $\Phi$ is finitely additive, nonatomic, and closed valued. Then

(1) the values of $\Phi$ are convex and $\overline{R \Phi}$ is convex;

(2) if $\mathscr{F}$ has Seever property, $R \Phi$ is convex; and

(3) if $\mathscr{F}$ has Seever property and $R \Phi \subseteq[0, \infty[$, then there exists $\mu \in S$ such that $R \Phi=[0, \mu(e)]=R \mu$.

Looking at the proof of [Dr, Theorem 6.1 and Corollary 6.2] in light of Proposition 4 we see that the hypothesis of $\sigma$-completeness of the algebra and the order continuity of the correspondence can be dropped in those results. So

Proposition 5. Let $X$ be a separated locally convex space. Assume $\Phi$ is a+finitely additive correspondence over the algebra $\mathscr{F}$. If $\Phi$ is $\sigma\left(X, X^{\prime}\right)$ nonatomic, then the values and the range of $\Phi$ have convex $\sigma\left(X, X^{\prime}\right)$-closures.

\section{REFERENCES}

[AR] T. E. Armstrong and M. K. Richter, The Core-Walras equivalence, J. Econom. Theory 33 (1984), 116-151.

[A] Z. Artstein, Set-Valued measures, Trans. Amer. Math. Soc. 165 (1972), 103-125.

[AB] A. Avallone and A. Basile, On the Liapunov-Richter theorem in the finitely additive setting, J. Math. Economics 22 (1993).

[B] A. Basile, Controls of families of finitely additive functions, Ricerche Mat. 35 (1986), 291-302.

[B1] - Finitely additive nonatomic coalition production economics: Core-Walrus equivalence, Internat. Econom. Rev. 34 (1993), 983-995.

[Bh] K. P. S. Bhaskara Rao and M. Bhaskara Rao, Theory of charges, Academic Press, New York, 1983.

[C] A. Costé, Sur les multimeasures á valeurs fermées bornées d'un espace de Banach, C. R. Acad. Sci. Paris Sér. I Math. 280 (1975), 567-570.

[C1] L La propriété de Radon-Nikodym en intégration multivoque, C. R. Acad. Sci. Paris Sér. I Math. 280 (1975), 1515-1518.

[C2] _- Densité des sélecteurs d'une multimesure à valeurs convexes fermées bornées d'un espace de Banach séparable, C. R. Acad. Sci. Paris Sér. I Math. 282 (1976), 967-969.

[D] G. Debreu, Integration of correspondences, Proc. Fifth Berkeley Sympos. Math. Stat. and Probability (1965/66), vol. II, Univ. of California Press, Berkeley, CA, 1967, pp. 351-372. 
[DS] G. Debreu and D. Schmeidler, The Radon-Nikodym derivative of a correspondence, Proc. Sixth Berkeley Sympos. Math. Stat. and Probability (1970), vol. II, Univ. of California Press, Berkeley, CA, 1972, pp. 41-56.

[DU] J. Diestel and J. J. Uhl, Vector measures, Math. Surveys Monographs, vol. 15, Amer. Math. Soc., Providence, RI, 1977.

[Dr] L. Drewnowski, Topological rings of sets, continuous set functions integration. I, II, III, Bull. Acad. Polon. Sci. Math. 20 (1972), 269-276, 277-286, 439-445.

[Dr1] L. Drewnowski, Additive and countably additive correspondences, Comment. Math. 19 (1976), 25-54.

[GT] C. Godet-Thobie, Multimesures et multimesures de transition, Thése, 1975.

[GT1] __ Some results about meultimeasures and their selectors, Proc. Conf. Oberwolfach 1979, Lecture Notes in Math., vol. 794, Springer-Verlag, New York, 1980, pp. 112-116.

[Gr] A. Grothendieck, Topological vector spaces, Gordon and Breach, New York, 1973.

[H] W. Hildenbrand, Core and equilibria of a large economy, Princeton Univ. Press, Princeton, NJ, 1974.

[J] R. C. James, Weakly compact sets, Trans. Amer. Math. Soc. 113 (1964), 129-140.

[KT] E. Klein and A. C. Thompson, Theory of correspondences. Including applications to mathematical economics, Wiley-Interscience, New York, 1984.

[L] Le Van Tu, The range of a multimeasure, J. Austral. Math. Soc. Ser. A 26 (1978), 442-452.

[P] R. Pallu de La Barriere, Quelques proprietes des multi-mesures, Seminaire d'Analyse Convexe Montpellier, Exposé n.11, 1973.

[Pr] A. M. Precupanu, On certain Nikodym type theorems for multimeasures, Ann. st. Univ. 'Al. I. Cuza' Iasi Mat. 35 (1989), 93-100.

[S] H. H. Schaefer, Topological vector spaces, Springer-Verlag, New York, 1971.

[Sc] D. Schmeidler, Convexity and compactness in countably additive correspondences, Differential Games and Related Topics (H. W. Kuhn and G. P. Szego, eds.), North-Holland, Amsterdam, 1971.

[Sc1] D. Schmeidler, On set correspondences into uniformly convex Banach spaces, Proc. Amer. Math. Soc. 34 (1972), 97-101.

[T] I. Tweddle, Weak compactness in locally convex spaces, Glasgow Math. J. 9 (1968), 123-127.

[V] K. Vind, Edgeworth allocations in exchange economy with many traders, Internat. Econ. Rev. 5 (1964), 165-177.

[VW] H. Volkmer and H. Weber, Der wetebereich atomloser inhalte, Arch. Math. 40 (1983), 464-474.

[W] H. Weber, Fortsetzung von massen mit werten in uniformen halbgruppen, Arch. Math. 27 (1976), 412-423.

[W1] _ Topological Boolean rings. Decomposition of finitely additive set functions, Pacific J. Math. 110 (1984), 471-495.

[W2] _ Group and vector valued s-bounded contents, Measure Theory Oberwolfach 1983, Lecture Notes in Math., vol. 1089, Springer-Verlag, New York, 1984.

[Z] Zhang Wenxiu, Ma Jifeng, and Li Aijie, The extension of a compact set-valued measure, J. Math. Res. Exposition 10 (1990), 35-43.

Dipartimento di Matematica e Applicazioni, Università Federico II, Complesso M. te S. ANGelo, via Cintia, 80126 Napoli, Italy 